\title{
THE CONDITION FOR AN ORTHONOMIC DIFFERENTIAL SYSTEM*
}

\author{
BY \\ JOSEPH MILLER THOMAS
}

This paper develops a method of testing whether a given finite set of partial derivatives can be placed in a specified order by assigning integral cotes to the independent variables and functions in Riquier's manner. $†$ The method gives a test for determining whether a system of partial differential equations is orthonomic, as far as the ordering of derivatives is concerned.

1 . Consider $r$ functions $u_{\alpha}$ of $n$ independent variables $x_{i}$. The partial derivative

$$
D_{i} u_{\alpha}=\frac{\partial^{i_{1}+\cdots+i_{n}} u_{\alpha}}{\partial x_{1}^{i_{1}} \cdots \partial x_{n}^{i_{n}}}
$$

is conveniently represınted by a matrix whose elements are integers and which has one row and $n+r$ columns, namely, by

$$
I=\left\|i_{1} i_{2} \cdots i_{n} 0 \cdots 1 \cdots 0\right\|,
$$

the element 1 being in the $(n+\alpha)$ th column.

Riquier's method of placing the partial derivatives of the functions $u$ in a definite order can be described as follows. Let there be given a fixed matrix $M$ whose elements are integers and whose rows are $n+r$ in number. The elements on the $a$ th column are called the $a$ th cotes of the variables $u, x$. Let $J$ be the matrix associated with another derivative, say with

$$
D_{j} u_{\beta}=\frac{\partial^{j_{1}+\cdots+j_{n}} u_{\beta}}{\partial x_{1}^{j_{1}} \cdots \partial x_{n}^{j_{n}}} .
$$

By definition the derivative $I$ follows or precedes $J$ according as the first nonzero element in the matrix (of one row)

$$
(I-J) M
$$

is positive or negative. This statement may be abbreviated symbolically by saying that $I$ follows or precedes $J$ according as

$$
(I-J) M>0 \text { or }(I-J) M<0 \text {. }
$$

* Presented to the Society, October 31, 1931; received by the editors in September, 1931.

† Riquier, C., Les Systèmes d'Equations aux Dérivées Partielles, Paris, 1910, p. 195. 
Suppose there are given certain order relations among a finite set of derivatives of the $u$ 's. Each of these order relations can be given the form " $I$ follows $J$." The problem of determining a matrix $M$ which establishes a given set of order relations among the derivatives can accordingly be phrased as follows: find a matrix of integers $M$ which satisfies the inequalities

$$
K M>0,
$$

where $K(=I-J)$ assumes a finite set of given values.

The conditions on the elements $\lambda_{1}$ of the first column of $M$ are

$$
\sum_{p=1}^{n+r} \lambda_{1}{ }^{p} k_{p} \geqq 0,
$$

where

$$
\begin{array}{rr}
k_{p}=i_{p}-j_{p} & (p=1,2, \cdots, n), \\
k_{n+\alpha}=1, k_{n+\beta}=-1 & (\alpha \neq \beta),
\end{array}
$$

and all the other $k$ 's are zero. If $\alpha=\beta$, then $k_{n+1}=\cdots=k_{n+r}=0$.

The discussion of system (1.6) is most readily accomplished, it seems, by adopting the geometric point of view employed by Miss Stokes.* To each of the given relations there corresponds a point in $(n+r)$-dimensional euclidean space, whose coördinates are $\left(k_{1}, k_{2}, \cdots, k_{n+r}\right)$. We shall speak of the set of points corresponding to the given order relations as the "set of representative points $S_{n+r}$." A solution of (1.6) is an oriented $(n+r-1)$-flat passing through the origin and separating no pair of representative points $k$.

There is a sub-set of $S_{n+r}$, called its inconsistent set, contained by every solution of (1.6). $\dagger$ An important property of the inconsistent set is given by

TheOREM 1. The system of inequalities

$$
\sum_{p=1}^{n+r} \lambda^{p} k_{p} \geqq 0
$$

whose coefficients are the coördinates of the points in any inconsistent set has only equality solutions, that is, solutions making all its left members zero.

To prove the above, we remark that the general solution of the system in question is given by $\ddagger$

\footnotetext{
* Stokes, R. W., A geometric theory of solution of linear inequalities, these Transactions, vol. 33 (1931), pp. 782-805.

† Stokes, loc. cit., p. 794. When we use the unqualified term "inconsistent set," we mean the case $l=0$, that is, the solution is not required a priori to contain any points of the original set. From (3.3) of that paper it is moreover clear that Theorem 9 is true whatever the rank of the system.

‡ Stokes, loc. cit., p. 786, formula (3.3).
} 


$$
\sigma+\sum_{i=q+1}^{n+r} a^{i} u_{i}
$$

where $q$ is the dimensionality of the flat space determined by the inconsistent 'set and the origin, the $u$ 's are expressions whose vanishing defines that flat space, and $\sigma$ is the general solution of the system determined by the point set in $q$ dimensions. From the definition of an inconsistent set, ${ }^{*}$ however, $\sigma=0$. Hence the $(n+r-1)$-flat (1.8) passes through all the points of the inconsistent set because the coördinates of those points make the $u$ 's zero. Consequently the only solutions of (1.7) are equality solutions, and the theorem is demonstrated.

In passing, it is perhaps worth while to mention the following corollary:

THEOREM 2. The inconsistent set of an inconsistent set of points is the set itself.

Returning to the discussion of the determination of a matrix satisfying (1.5), we see that the second $\operatorname{cotes} \lambda_{2}^{p}$ must satisfy

$$
\sum_{p=1}^{n+r} \lambda_{2}^{p} k_{p} \geqq 0,
$$

where $\left(k_{1}, \cdots, k_{n+r}\right)$ ranges over the inconsistent set of $S_{n+r}$. This is true because any solution of (1.6) passes through the inconsistent set of $S_{n+r}$, that is, the left members of (1.6) which correspond to the inconsistent-set are zero for every solution of (1.6). The first elements in the corresponding $K M$ 's being zero, it is necessary that the second elements be non-negative.

Theorem 1 shows that (1.9) has only equality solutions. The third cotes must therefore satisfy the same system as the second. This statement is true of all succeeding cotes. Consequently we have

$$
K M=0,
$$

where $K$ ranges over the inconsistent set, no matter how many columns of $M$ are determined to satisfy (1.6) and the analogous succeeding conditions.

If the inconsistent set actually contains one or more points, it is therefore impossible to determine an $M$ placing the derivatives in the specified order.

If the inconsistent set is vacuous, the associated system

$$
\sum_{p=1}^{n+r} \lambda_{1}^{p} k_{p}>0
$$

obtained from (1.6) by replacing the sign $\geqq$ by $>$, has a solution. $\dagger$

* Stokes, loc. cit., p. 794, for $l=0$.

† Stokes, loc. cit., p. 794, Theorem 10 for $l=0$. 
When the rank of (1.10) is $n+r$, its general solution is a linear homogeneous combination of the complete system of fundamental solutions of (1.6), the coefficients being arbitrary positive constants. ${ }^{*}$ A fundamental solution is obtained from the given numbers $k$ by rational operations, and is therefore composed of rational numbers, when the $k$ 's are integral, as they are here. The arbitrary constant, which multiplies any fundamental solution of (1.6) as it appears in the general solution of (1.10), can be chosen so as to remove the denominator occurring in the fundamental solution. Hence, if (1.10) has a solution, it has a solution in integers.

The case where the rank of (1.10) is less than $n+r$ can be reduced to the case already treated. $\dagger$ The result just established therefore holds in general.

We have accordingly shown that if a matrix establishing the given order relations exists, system (1.10) has a solution in integers $\lambda_{1}$. A matrix with a single column composed of these $\lambda$ 's will effect the given ordering. Hence we have

THEOREM 3. If a finite set of order relations can be established by a matrix of integers, it can be established by a matrix of integers having a single column. $\ddagger$

As a consequence of this and of the geometric condition $\S$ that (1.10) have a solution we get

THEOREM 4. A given finite set of order relations can be effected by a matrix of integers if and only if the origin is exterior to the convex figure determined by the representative points.

Analytic methods for testing data and for finding the $\lambda$ 's when they exist are to be found in Miss Stokes' paper.

2. Riquier $\|$ finds it desirable to make the first cotes of the independent variables unity, i.e., to make the first $n$ elements on the first column of $M$ equal to unity. If we let

$$
k=i_{1}+\cdots+i_{n}-j_{1}-\cdots-j_{n},
$$

the remaining first $\operatorname{cotes} \mu^{n+1}, \cdots, \mu^{n+r}$ must satisfy

* Stokes, loc. cit., p. 793, Theorem 8.

† Stokes, loc. cit., p. 786. If the $a$ 's in (3.3) are made zero, it is clear that the general solution in the subspace is a particular solution of the original system.

$\ddagger$ The theorem is not true in general if the set of order relations is infinite. For example, a matrix $M$ ordering all derivatives must contain at least $n$ columns, where $n$ is the number of independent variables. Cf. a paper by the author, Matrices of integers ordering derivatives, these Transactions, vol. 33 (1931), p. 393.

$\S$ Stokes, loc. cit., p. 804, Theorem 16.

|| Loc. cit., p. 207, footnote 2. 


$$
k+\sum_{p=n+1}^{n+r} \mu^{p} k_{p} \geqq 0 .
$$

This system has a solution if and only if the homogeneous system

$$
\mu k+\sum_{p=n+1}^{n+r} \mu^{p} k_{p} \geqq 0, \mu>0,
$$

has a solution.*

The points $\left(k, k_{n+1}, \cdots, k_{n+r}\right)$ plus the point $(1,0, \cdots, 0)$ will be referred to as the "set $S_{r+1}$ of representative points in $r+1$ dimensions" just as the set previously described is the "set $S_{n+r}$ of representative points in $n+r$ dimensions." System (2.2) has a solution if and only if

$$
\mu k+\sum_{p=n+1}^{n+r} \mu^{p} k_{p} \geqq 0, \mu \geqq 0,
$$

has a solution not containing the point $(1,0, \cdots, 0)$. Since the general solution of (2.3) contains the inconsistent set of $\delta_{r+1}$, it is necessary that $(1,0, \cdots, 0)$ be not in that inconsistent set. We suppose this condition fulfilled.

The only points of $\delta_{r+1}$ which are in all the fundamental solutions of (2.3) are those of its inconsistent set. $\dagger$ Hence by giving positive values to the arbitrary constants in the general solution of (2.3) we get a solution of (2.2) which contains no point of $S_{r+1}$ except those in its inconsistent set.

Let the points of $S_{n+r}$ which correspond to points in the inconsistent set of $S_{r+1}$ be called the derived set of $S_{n+r}$ and denoted by $S_{n+r}^{\prime}$. When the first cotes have the values whose determination was indicated above, in (1.6) the sign $=$ holds for points of $S_{n+r}^{\prime}$ and the sign $>$ for all other points. Hence the only conditions to be satisfied by the second cotes are (1.9) in which $k$ ranges over the set $S_{n+r}^{\prime}$. The discussion concerning the existence of $\lambda_{1}$ 's satisfying (1.6) in the proof of Theorem 3 applies verbatim.

As before, the operations in solving are rational. The elements of $M$ can be rendered integral by multiplying them by a properly chosen positive integer. Hence we have the following results.

THEOREM 5. If a given finite set of order relations among derivatives can be established by means of a matrix of inlegers, the first cote of each independent variable being unity, it can be established by a matrix of two columns.

* Stokes, loc. cit., \$13.

$\dagger$ This result follows readily from Theorem 11 of Miss Stokes' paper. 
THEOREM 6. $A$ given finite set of order relations can be effected by a matrix of integers having unity as the first cote of each independent variable if and only if the point $(1,0, \cdots, 0)$ is not in the inconsistent set for $r+1$ dimensions and the origin is exterior to the convex figure determined by the derived set of points in $n+r$ dimensions.

The above method can be employed to obtain a necessary and sufficient condition for the existence of a matrix which establishes a given set of order relations and which has any set of its elements given.

3. In applying the foregoing results, the dimensionality of the spaces considered can be diminished by unity in the following manner. The elements of $M$ corresponding to any function $u$, that is, the elements on any one of the last $r$ rows, can be made zero by a transformation of $M$ which preserves order.* Hence, in particular, the elements in the last row of $M$ can be assumed as zero, and consequently the last coördinate of the points in $S_{n+r}$ and $\mathcal{S}_{r+1}$ ignored.

4. The chief application of the above is in determining whether a given system of partial differential equations is orthonomic. $\dagger$ To do this, it is necessary to determine whether there is a matrix of integers placing the derivatives in such an order that all the derivatives appearing in any given right member precede the derivative which constitutes the corresponding left member.

This application will now be illustrated by two examples. First consider the single equation $\ddagger$

$$
\frac{\partial^{2} u}{\partial x \partial y}=F\left(\frac{\partial^{2} u}{\partial x^{2}}, \frac{\partial^{2} u}{\partial y^{2}}\right)
$$

In order that the system be orthonomic, it is necessary that

$$
\frac{\partial^{2} u}{\partial x \partial y}>\frac{\partial^{2} u}{\partial x^{2}}, \quad \frac{\partial^{2} u}{\partial x \partial y}>\frac{\partial^{2} u}{\partial y^{2}},
$$

the sign $>$ being read "follows." These conditions are represented geometrically by the points

$$
\begin{aligned}
& S_{3}:(-1,1,0),(1,-1,0) ; \\
& S_{2}:(0,0),(0,0),(1,0) .
\end{aligned}
$$

The inconsistent set of $S_{2}$ is thus $(0,0)$, and $S_{3}^{\prime}$ coincides with $S_{3}$. The convex figure determined by $S_{3}^{\prime}$ is a line segment containing the origin. Hence (4.1)

\footnotetext{
* Thomas, loc. cit., p. 391.

† Riquier, loc. cit., p. 201.

† Riquier, loc. cit., p. xx.
} 
is not orthonomic. It will become orthonomic if either derivative is removed from its right member.

As a second example consider the system

$$
\begin{gathered}
\frac{\partial u}{\partial x}=f\left(\frac{\partial^{2} v}{\partial y^{2}}, v\right), \\
\frac{\partial^{3} v}{\partial x^{2} \partial y}=g\left(\frac{\partial^{2} u}{\partial y^{2}}, \frac{\partial^{2} u}{\partial x \partial y}\right) .
\end{gathered}
$$

The representative points are

$$
\begin{aligned}
& S_{4}:(1,-2,1,-1),(1,0,1,-1),(2,-1,-1,1),(1,0,-1,1) ; \\
& S_{3}:(-1,1,-1),(1,1,-1),(1,-1,1),(1,-1,1),(1,0,0) .
\end{aligned}
$$

Ignoring the last coördinate, we find the inconsistent set of $S_{3}$ to be $(-1,1)$ and $(1,-1)$. Since $(1,0)$ is not in the inconsistent set, the first condition of Theorem 6 is satisfied. Furthermore, the derived set $S_{4}^{\prime}$ is seen to be

$$
S_{4}^{\prime}:(1,-2,1),(2,-1,-1),(1,0,-1) \text {. }
$$

The origin is exterior to the convex figure determined by these three points. Hence system (4.2) is orthonomic, as far as ordering of derivatives is concerned. A matrix putting the derivatives in the desired order is

DUKe UNIVERSITY,

$$
M=\left\|\begin{array}{ll}
1 & 1 \\
1 & 0 \\
1 & 0 \\
0 & 0
\end{array}\right\|
$$

DURzaM, N. C. 